OPEN ACCESS

Edited by:

Marina I. Arleevskaya, Kazan State Medical Academy,

Russia

Reviewed by:

Yves Renaudineau,

Brest University Medical School,

France

Ana Claudia Zenclussen, Otto-von-Guericke University,

Germany

Julia Simard,

Stanford University, USA

${ }^{*}$ Correspondence:

Christine G. Parks

parks1@mail.nih.gov

Specialty section: This article was submitted to Microbial Immunology, a section of the journal

Frontiers in Immunology

Received: 08 January 2016 Accepted: 07 March 2016

Published: 31 March 2016

Citation:

Parks CG, D'Aloisio AA and Sandler DP (2016) Early Life Factors

Associated with Adult-Onset Systemic Lupus

Erythematosus in Women.

Front. Immunol. 7:103.

doi: 10.3389/fimmu.2016.00103

\section{Early Life Factors Associated with Adult-Onset Systemic Lupus Erythematosus in Women}

\author{
Christine G. Parks ${ }^{1 *}$, Aimee A. D'Aloisio ${ }^{1,2}$ and Dale P. Sandler ${ }^{1}$ \\ ${ }^{1}$ Epidemiology Branch, National Institute of Environmental Health Sciences, Research Triangle Park, Durham, NC, USA, \\ ${ }^{2}$ Social \& Scientific Systems, Inc., Durham, NC, USA
}

Background: Exposure early in life can influence adult disease and immunity, but the role of early life exposures in risk of systemic lupus erythematosus (SLE) is not established.

Methods: Women in a national cohort (ages 35-74) provided data on perinatal, maternal, and sociodemographic factors, longest residence to age 14, and residential farm history of at least 12 months to age 18. Cases $(N=124)$ reported SLE diagnosed age 16 years or older with use of disease modifying antirheumatic drugs. Non-cases $(N=50,465)$ did not report lupus. Odds ratios (OR) and 95\% confidence intervals $(\mathrm{Cl})$ were estimated by logistic regression adjusting for age and race/ethnicity.

Results: SLE was associated with low birthweight (data on 84 cases and 36,477 noncases; $<2,500$ versus 3,000 to $<3,500 \mathrm{~g} \mathrm{OR}=2.2 ; 95 \% \mathrm{Cl} 1.2,3.9$ ) and preterm birth (57 cases and 22,784 non-cases; $\geq 1$ month early versus full-term $\mathrm{OR}=3.4 ; 95 \% \mathrm{Cl}$ $1.6,7.4)$. Considering longest childhood residence to age 14, SLE was associated with more frequent pesticide use (e.g., at least monthly $\mathrm{OR}=2.3 ; 95 \% \mathrm{Cl} 1.3,4.1$ ). SLE was associated with having an early and extended childhood farm residence (i.e., prenatal/ maternal farm exposure and longest childhood farm residence $\mathrm{OR}=1.8 ; 95 \% \mathrm{Cl} 1.1$, 3.0 versus neither). In those with a childhood-only farm residence of $12+$ months, agricultural pesticide use was associated with SLE, with the strongest associations for direct personal exposures.

Conclusion: The association of SLE with preterm birth is consistent with studies in other populations and with an observed association with low birthweight. The associations of SLE with childhood exposure to residential and agricultural pesticides warrant further study.

Keywords: lifecourse epidemiology, pesticide exposure, birthweight, preterm birth, autoimmune disease

\section{INTRODUCTION}

Systemic lupus erythematosus (SLE) is an autoimmune disease characterized by immune reactivity to multiple nuclear components and inflammation, resulting in diverse clinical features and multiple organ involvement. The causes of SLE are generally not known. Racial disparities and increased familial risk suggest a genetic predisposition. It is believed that environmental factors may contribute 
to the development of disease, but knowledge on specific risk factors is mostly limited to occupational exposures (1).

The developmental origins hypothesis has been proposed for many adult-onset, chronic inflammatory diseases, including SLE (2-6). Exposures during and after gestation, including nutritional, infectious, chemical, and psychosocial factors (7-11), play a critical role in shaping future immunity. A few epidemiologic studies have examined perinatal and childhood factors associated with SLE, with results including evidence of an association with preterm birth and inconsistent associations with birthweight $(12,13)$.

Socioeconomic factors have been associated with disease severity, inflammation, and adverse outcomes in $\operatorname{SLE}(14,15)$. Although socioeconomic disparities originating from childhood have been described across a variety of chronic inflammatory diseases (16), the role of childhood socioeconomic status (SES) and related adversities in risk of SLE is not known. Other early life immune modifying exposures (e.g., infections, vitamin D) have also been considered in relation to SLE risk (17).

History of farm occupation and pesticide exposures has been associated with SLE (18-20), but the role of childhood exposures have not been specifically examined. One past study suggested an association of SLE with mixing pesticides in a farm environment (21), while another showed a dose-response association personal pesticide in women use with rheumatoid arthritis (RA) and SLE that was highest in women with a farm history (18). Women who live or work on a farm as adults may be more likely to have grown up on a farm. Therefore, it is plausible that previous studies showing SLE associations with farm-related pesticide use may reflect to some extent an association with childhood exposures.

In this study, we examine associations between adult-onset SLE in a national cohort of women and a range of perinatal and early life exposures, some of which had previously been associated with RA in this same cohort (22). Investigating the role of early life factors in risk of SLE will help us to understand what factors underlie susceptibility for developing autoimmune disease in adulthood. Along with knowledge of genetic susceptibility, understanding the developmental origins of SLE risk may help identify opportunities for prevention and mechanisms contributing to immune dysregulation.

\section{MATERIALS AND METHODS}

\section{Sample and Case Identification}

We conducted a case-control analysis using baseline data from the NIEHS Sister Study cohort $(N=50,884$ women ages $35-74$, enrolled 2003-2009). Women were eligible if they had a sister with breast cancer, but did not have breast cancer themselves at enrollment (23). Participants were identified through self-referral following a broad advertising campaign in the U.S. and Puerto Rico. The Sister Study was approved by the Institutional Review Board of the National Institute of Environmental Health Sciences, NIH. Participants provided written informed consent.

Probable cases $(n=124)$ were classified based on a self-reported doctor's diagnosis of SLE at age 16 or older and reported use of disease modifying antirheumatic drugs (DMARDs). Cases with missing data on medications or not reporting DMARDs $(N=153)$, reporting non-specified or discoid lupus only $(N=61)$, or diagnosis before age 16 were excluded $(n=6)$. The most common DMARDs reported by probable cases were hydroxychloroquine ( $90 \%$ ever and $70 \%$ currently used) and methotrexate (11\% ever and $10 \%$ current). No data on disease severity or nephritis were available; however, medications often used to treat nephritis were less common (e.g., Azothioprine; $8 \%$ ever used). Five probable cases (5\% of $124)$ also reported a diagnosis and DMARD use for RA. Non-cases $(n=50,465)$ reported no lupus. Other possible cases of autoimmune disease were not excluded from the referent, but are unlikely to influence results due to their relative rarity in the overall sample.

\section{Exposures and Covariates}

Computer-assisted telephone interviews collected data on sociodemographic factors, childhood residential history, lifestyle factors, personal medical history, and medication use. Questions on sociodemographic factors included food insecurity ("When you were growing up, were there times your family didn't have enough to eat?"), highest household education level when respondent was age 13, and relative household income while growing up (well off, middle income, low income, or poor). Adult sociodemographic covariates included participant age (baseline), race/ethnicity (dichotomized for this analysis as non-Hispanic White and nonWhite, including black, Hispanic, and other racial and ethnic groups), and education level (dichotomized for this analysis as less than a college degree, including $<12$ years, high school degree or equivalent, some college but no degree, or associate degree/ technical degree versus college degree, including college graduate/bachelor's degree and graduate/professional degree).

Data on the longest childhood residence to age 14 years and childhood farm residence of 12 months or longer to age 18 were collected by computer-assisted telephone questionnaire. For the longest childhood residence, participants born in the U.S. were grouped by state into: (1) census regions (West, Midwest, Northeast, South, and Puerto Rico) and (2) stratified by 40th and 35th parallels (approximate according to U.S. state). Questions about the longest childhood residence included whether the residence was a farm, had been a farm, or was near a farm, and whether pesticides were used in and around the house or garden, including frequency of use. Women who did not report a farm as longest childhood, adult, or current residence were also asked whether they had lived on any other farm for 12 or more months. A detailed questionnaire then asked about pesticide use on crops and animals, including opportunities for personal exposures (i.e., personally mixed or applied, and presence in the field when pesticides were being sprayed) for all residential farm experience 12 months or more up to age 18. Similar questions were asked for residential and agricultural pesticides based on current and longest adult residence and any farm residence 12 months or more ages 18 years and older.

Participants were also mailed family history questionnaires that included assessment of perinatal factors and were encouraged to contact their mother or other relatives to assist with as needed to provide data on maternal age at participants' birth, prenatal, maternal, and household smoking, maternal farm residence or work on farm while pregnant, breast feeding, birthweight (pounds, ounces), and gestational age at birth. Participants unable to report exact birthweight were asked whether birthweight was 
$<5$ pounds or at least 5 pounds, and responses were converted to grams and categorized $(<2,500 ; 2,500-3,499 ; 3,400-3,999$; and $\geq 4,000 \mathrm{~g}$ ).

Birth order was calculated from brother birthdates reported in the family history questionnaires and sister birthdates reported during the computer-assisted telephone interviews. Other perinatal factors [multiple birth, soy formula, maternal/ gestational diabetes, gestational hypertension or preeclampsia, and diethylstilbestrol (DES) exposure] were not considered due to low prevalence.

\section{Analyses}

Analyses were conducted in SAS (version 9.2, SAS Institute Inc., Cary, NC, USA). Logistic regression was used to estimate odds ratios (OR) and 95\% confidence intervals (CI). Significant findings were those in which the $95 \% \mathrm{CI}$ excluded the null $(\mathrm{OR}=1.0)$, corresponding to a two-sided $p<0.05$. Initial models were ageadjusted and secondary models also adjusted for race/ethnicity. We also evaluated associations with a childhood SES adversity score [a sum of four factors - young maternal age at birth ( $<20$ years), low or poor relative childhood household income, maximum household education less than college, and childhood food insecurity], which was previously examined with respect to RA in this cohort (22). For significant associations, we examined possible confounding by education and conducted sensitivity analyses excluding non-Whites (a higher risk subgroup and potential effect modifier) and women over age 60 (with higher potential for errors in reporting of perinatal and childhood factors). Results were not substantially changed unless otherwise noted. Data on adult factors (education, residential pesticides, and farm residence) were used to explore potential joint effects and identify independent "childhood only" exposures.

\section{RESULTS}

Cases were slightly younger than non-cases and enrolled in the cohort within a median of 11 years since diagnosis (Table 1).
Cases were more likely than non-cases to be of non-White race/ ethnicity (including black or Hispanic) and were less likely to have graduated from college.

Systemic lupus erythematosus was associated with low birthweight, with a linear dose response $(p$-trend $=0.008)$ and with preterm (at least 1 month early) versus term birth (Figure 1). Excluding preterm births (seven of eight preterm cases also had a low birthweight), the overall trend was no longer significant ( $p=0.08$; not shown); however, when the sample was restricted to women under age 60 , the trend across low birthweight categories became significant again ( $p=0.03$, not shown). For women providing exact birthweight, cases $(N=84)$ weighed 6.8 (SD 1.6) pounds at birth compared with 7.2 (SD 1.3) in non-cases $(N=n=36,477)$, for an adjusted $\mathrm{OR}=0.80 ; 95 \% \mathrm{CI} 0.69,0.94$ per pound.

TABLE 1 | Characteristics of probable SLE cases and non-cases in the Sister Study.

\begin{tabular}{lcc}
\hline & $\begin{array}{c}\text { Probable SLE } \\
\mathbf{N}=\mathbf{1 2 4}\end{array}$ & $\begin{array}{c}\text { Non-cases } \\
\mathbf{N}=\mathbf{5 0 , 4 6 5}\end{array}$ \\
\cline { 2 - 3 } & \multicolumn{2}{c}{ Median years (IQR) } \\
\hline Age at enrollment & $54(47,60)$ & $55(60,61)$ \\
Age at diagnosis & $43(33,50)$ & $\mathrm{N} / \mathrm{A}$ \\
Time since diagnosis & $11(3,16)$ & $\mathbf{N} / \mathrm{A}$ \\
\hline & $\mathbf{N}(\%)$ & $\mathbf{N ~ ( \% )}$ \\
\hline Race/ethnicity & & \\
$\quad$ White & $86(69)$ & $42,269(84)$ \\
$\quad$ Non-White & $38(31)$ & $8,181(16)$ \\
Educational attainment & & \\
SHigh school degree & $16(13)$ & $7,725(15)$ \\
Some college or technical degree & $59(48)$ & $17,010(34)$ \\
4-year college graduate & $25(20)$ & $13,633(27)$ \\
Professional or graduate degree & $24(19)$ & $12,085(24)$
\end{tabular}

aNon-Hispanic White and non-White (Black - 19\% of cases versus $9 \%$ of noncases, Hispanic $9 \%$ of cases versus $5 \%$ of non-cases, and other racial and ethnic groups - $3 \%$ cases and non-cases).

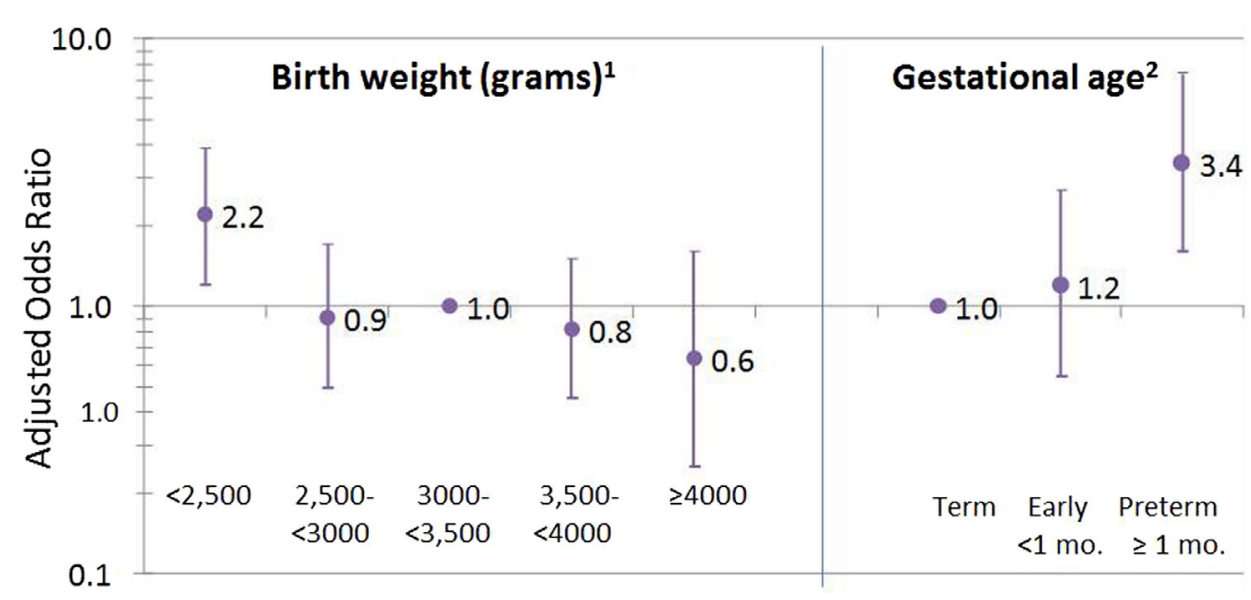

FIGURE 1 | Birthweight and gestational age associated with SLE. ${ }^{1}$ Missing on 39 cases (31\%) and 13,659 (27\%) non-cases; test for trend across birthweight categories, $p=0.008$. ${ }^{2}$ Missing or do not know for 67 cases (54\%) and 27,681 (55\%) non-cases. 
Systemic lupus erythematosus cases were significantly more likely to have been born to mothers who lived or worked on a farm while pregnant (Table 2). Birth season, prenatal smoking (maternal and household), being breastfed, firstborn, and sibling number were not significantly associated with SLE. Overall, SLE was not associated with individual childhood social factors. We saw no evidence of joint effects for combined lower adult educational attainment and increased SES score (Table S1 in Supplementary Material); in college educated women odds of

TABLE 2 | Perinatal and childhood sociodemographic risk factors for SLE.

Case Non-case Odds ratios ${ }^{\mathrm{a}}(95 \% \mathrm{Cl}$; $N=124 n=50,465$ confidence intervals)

$N(\%) \quad N(\%)$

Birth characteristics and perinatal factors

Mom lived or worked on farm

while pregnant

\begin{tabular}{|c|c|c|c|}
\hline No & $88(75)$ & $39,560(82)$ & Referent \\
\hline Yes & $30(25)$ & $8,803(18)$ & $1.6(1.0,2.4)^{b}$ \\
\hline \multicolumn{4}{|l|}{ Prenatal smoking } \\
\hline None & $35(32)$ & 15,303 (34) & Referent \\
\hline Mom only & $9(8)$ & $3,406(8)$ & $1.2(0.58,2.5)$ \\
\hline Household only & $45(41)$ & 14,917 (34) & $1.3(0.86,2.1)$ \\
\hline Both & 21 (19) & $10,853(24)$ & $0.90(0.53,1.6)$ \\
\hline \multicolumn{4}{|l|}{ Birth season } \\
\hline Winter (December-February) & $29(24)$ & $12,214(24)$ & $1.3(0.76,2.2)$ \\
\hline Spring (March-May) & $32(26)$ & $12,157(24)$ & $1.4(0.83,2.2)$ \\
\hline Summer (June-August) & 24 (19) & $13,170(26)$ & Referent \\
\hline Fall (September-November) & $39(31)$ & $12,924(26)$ & $1.6(0.93,2.6)$ \\
\hline \multicolumn{4}{|l|}{ Breastfed } \\
\hline No & $61(54)$ & $22,526(49)$ & Referent \\
\hline Yes & $51(46)$ & 23,339 (51) & $1.2(0.84,1.8)$ \\
\hline \multicolumn{4}{|l|}{ Firstborn } \\
\hline No & $98(81)$ & $37,936(77)$ & Referent \\
\hline Yes & $23(19)$ & $11,412(23)$ & $0.90(0.57,1.4)$ \\
\hline \multicolumn{4}{|l|}{ Sibling number ${ }^{c}$} \\
\hline One & $16(13)$ & $6,091(12)$ & $1.2(0.71,2.2)$ \\
\hline 2 or 3 & $48(40)$ & $21,824(44)$ & Referent \\
\hline 4 or 5 & $28(23)$ & $12,181(25)$ & $0.95(0.60,1.5)$ \\
\hline 6 or more & $29(24)$ & $9,515(19)$ & $1.1(0.70,1.8)$ \\
\hline \multicolumn{4}{|l|}{ Early life social factors ${ }^{d}$} \\
\hline Young maternal age & $11(9)$ & $2,358(5)$ & $1.7(0.91,3.2)$ \\
\hline Lower income & $46(37)$ & $17,044(34)$ & $1.0(0.71,1.5)$ \\
\hline Lower household education & $72(60)$ & $27,041(54)$ & $1.1(0.79,1.7)$ \\
\hline Food insecurity & $15(12)$ & $4,753(12)$ & $1.1(0.64,1.9)$ \\
\hline \multicolumn{4}{|l|}{ SES adversity factors ${ }^{e}$} \\
\hline 0 & 34 (29) & $17,643(36)$ & 1.0 (referent) \\
\hline 1 & $40(34)$ & 16,319 (34) & $1.2(0.78,2.0)$ \\
\hline 2 & $33(29)$ & $10,817(22)$ & $1.4(0.90,7.3)$ \\
\hline $3-4$ & $11(9)$ & $3,613(7)$ & $1.2(0.61,2.5)$ \\
\hline
\end{tabular}

${ }^{a}$ Odds ratios and $95 \%$ confidence intervals adjusted for age and race/ethnicity. ${ }^{b} p$-Value $<0.05$.

'Women must have a sister to be enrolled in study. Thus, all have at least one sibling. 'Young maternal age of $<20$ years of age at birth, relative household income reported as low income or poor, and maximum household education in adults/parents less than a college degree.

eSocioeconomic status (SES) summary score based across four variables - young maternal age, lower household income and education, and food insecurity; $p$-trend $=0.26$.
SLE were almost doubled for women with two or more childhood SES adversity factors compared with only one or none $(\mathrm{OR}=2.0$; 95\% CI 1.2, 3.6).

For longest childhood residence, apparent geographical differences in SLE by census region or latitude (a higher prevalence of SLE cases than non-cases in the South and lower latitude) were non-significant after adjusting for race/ethnicity (Table 3). Odds of SLE were somewhat elevated for living in a rural compared with a suburban environment; however, when compared with all other areas combined, the association of SLE with rural residence was significant $(\mathrm{OR}=1.6 ; 95 \% \mathrm{CI} 1.1,2.3)$. An association was seen for SLE with use of residential pesticides in childhood, with a dose response for more frequent use (none, less than once per month, or at least once per month; $p$-trend $<0.0001$ ). This association was not confounded by adult residential pesticide

TABLE 3 | Characteristics of longest childhood residence and associations with SLE.

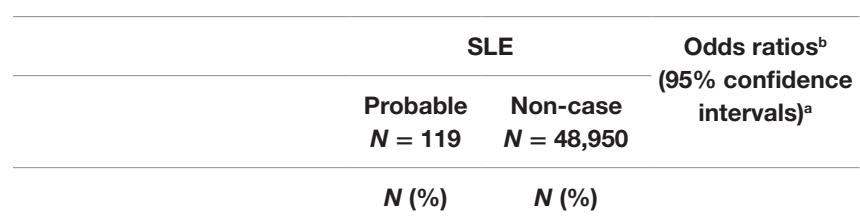

\begin{tabular}{|c|c|c|c|}
\hline \multicolumn{4}{|l|}{ Longest childhood residence ${ }^{a}$} \\
\hline \multicolumn{4}{|l|}{ U.S. Region } \\
\hline West & $18(15)$ & $7,032(14)$ & $1.3(0.70,2.3)$ \\
\hline Midwest & $32(27)$ & $17,443(36)$ & Referent \\
\hline Northeast & $30(24)$ & $12,087(25)$ & $1.4(0.83,2.2)$ \\
\hline South & $36(29)$ & $11,428(23)$ & $1.4(0.85,2.3)$ \\
\hline Puerto Rico & $3(3)$ & $915(2)$ & $\mathrm{NC}$ \\
\hline \multicolumn{4}{|l|}{ Latitude } \\
\hline$\leq 35^{\circ}$ & $29(24)$ & $7,918(16)$ & Referent \\
\hline $35-40^{\circ}$ & $30(25)$ & $11,461(12)$ & $0.90(0.52,1.5)$ \\
\hline$>40^{\circ}$ & $60(50)$ & $29,526(60)$ & $0.75(0.46,1.2)$ \\
\hline \multicolumn{4}{|l|}{ Environment } \\
\hline Urban & $26(21)$ & $11,324(23)$ & $0.83(0.49,1.4)$ \\
\hline Suburban/other & $33(27)$ & $13,927(27)$ & Referent \\
\hline Small town & $24(20)$ & $11,663(23)$ & $0.79(0.47,1.3)$ \\
\hline Rural & $39(32)$ & $12,851(20)$ & $1.4(0.86,2.0)$ \\
\hline \multicolumn{4}{|l|}{ Residential pesticides ${ }^{\mathrm{C}}$} \\
\hline Never used & $70(61)$ & $34,500(79)$ & Referent \\
\hline Infrequent (<monthly) & $29(25)$ & $7,657(17)$ & $1.6(1.0,2.5)$ \\
\hline Frequent (monthly+) & $15(13)$ & $2,534(6)$ & $2.3(1.3,4.1)$ \\
\hline \multicolumn{4}{|l|}{ Residence } \\
\hline Was not a farm & $67(58)$ & $28,960(64)$ & Referent \\
\hline Near a farm & $15(13)$ & $6,213(14)$ & $1.1(0.65,2.6)$ \\
\hline Used to be a farm & $10(9)$ & $2,971(7)$ & $1.6(0.80,3.0)$ \\
\hline Was a farm & $24(21)$ & $7,093(16)$ & $1.6(1.0,2.6)^{c}$ \\
\hline \multicolumn{4}{|c|}{ Early life and extended childhood farm residence ${ }^{d}$} \\
\hline No maternal or childhood & $81(74)$ & $33,272(77)$ & Referent \\
\hline Only maternal/prenatal & $6(5)$ & $3,293(8)$ & $0.70(0.30,1.6)$ \\
\hline Only childhood & $3(3)$ & $1,869(4)$ & $\mathrm{NC}$ \\
\hline Both maternal and childhood & $20(18)$ & $4,963(11)$ & $1.8(1.1,3.0)$ \\
\hline
\end{tabular}

${ }^{a}$ Residence location was missing or outside the U.S. for 5 cases and 1,560 non-cases. Women were grouped by state into census regions and by latitude based on majority of state area and/or population.

${ }^{b}$ Odds ratios calculated by logistic regression, adjusted for age and race/ethnicity; not calculated (NC) if 3 or fewer cases.

U Use by self or others; $p$-trend $=0.002$.

'Mother lived or worked on a farm while pregnant and longest childhood residence was a farm; referent group includes women who lived near a farm or on a former farm and women with other short-term farm experiences. 
use (reported for current or longest held adult residence by $55 \%$ of cases and $45 \%$ of non-cases), even though use of childhood residential pesticides was more common in women who reported adult residential pesticides (not shown). Compared to women who did not report any residential pesticide use, SLE was significantly associated with having used pesticides in both childhood and as an adult $(\mathrm{OR}=1.8 ; 95 \% \mathrm{CI} 1.1,3.1)$ and a non-significant association of similar magnitude was seen for childhood-only use $(\mathrm{OR}=1.6 ; 95 \% \mathrm{CI} 0.88,2.9)$ (Table S2 in Supplementary Material), supporting an independent association with childhood-pesticide use regardless of adult use.

While having a long-term childhood farm residence was associated with SLE, when coupled with information on maternal farm history, this reflected the association with an early and extended farm experience (i.e., prenatal farm exposure and longest childhood farm residence was a farm OR $=1.8 ; 95 \%$ CI 1.1 , 3.0) (Table 3). Few cases $(n=3)$ reported having their longest childhood residence on a farm in the absence of maternal/prenatal farm experience, and maternal/prenatal farm exposure alone was not associated with SLE. Based on data from the more detailed farm residence questionnaire, SLE was associated with living on a farm at least 12 months in childhood-only, but not as an adult (Table 4). In this context, SLE was associated with self-reported personal exposure to pesticides in childhood through application to crops. An elevated, non-significant association was seen for use on animals, and a stronger association was seen for exposure to pesticides from both animals and crops compared to either one alone. For those women with data on both longest childhood farm residence and agricultural pesticide use, the majority (80\%) of cases and (70\%) non-cases who reported agricultural pesticides

TABLE 4 | Farm residence and agricultural pesticide use associated with SLE.

\begin{tabular}{|c|c|c|c|}
\hline & \multicolumn{2}{|c|}{ SLE } & \multirow{3}{*}{$\begin{array}{c}\text { Odds ratios }^{a} \\
\text { (95\% confidence } \\
\text { intervals) }\end{array}$} \\
\hline & Probable & Non-case & \\
\hline & $\begin{array}{c}N=124 \\
N(\%)\end{array}$ & $\begin{array}{c}N=50,465 \\
N(\%)\end{array}$ & \\
\hline \multicolumn{4}{|l|}{ Lived on farm $12+$ months } \\
\hline Never & $87(72)$ & $37,288(77)$ & Referent \\
\hline Childhood only & $28(23)$ & $7,348(15)$ & $1.7(1.1,2.7)$ \\
\hline Child and adulthood & $4(3)$ & $2,275(5)$ & $0.85(0.31,2.3)$ \\
\hline Adult only & $2(2)$ & $1,757(4)$ & $0.57(0.14,2.3)$ \\
\hline \multicolumn{4}{|c|}{ Childhood-only farm pesticide exposures versus never lived on farm } \\
\hline Never lived on farm & $87(76)$ & $37,288(85)$ & Referent \\
\hline \multicolumn{4}{|l|}{ Farmed - pesticides on crops } \\
\hline None used & $8(7)$ & $3,132(7)$ & $1.2(0.58,2.5)$ \\
\hline No personal exposures & $6(5)$ & $2,479(6)$ & $1.1(0.47,2.5)$ \\
\hline Personal exposures & $13(11)$ & $1,288(3)$ & $4.2(2.4,7.7)$ \\
\hline \multicolumn{4}{|l|}{ Farmed - animals } \\
\hline No livestock contact & $7(6)$ & $2,184(5)$ & $1.4(0.66,2.9)$ \\
\hline Contact, no pesticides used & $13(11)$ & $3,676(8)$ & $1.5(0.90,2.6)$ \\
\hline Contact, pesticides used & $7(6)$ & $1,118(3)$ & $2.1(0.98,4.6)$ \\
\hline \multicolumn{4}{|l|}{ Personal pesticide exposures } \\
\hline None & 7 (6) & $2,744(6)$ & $1.2(0.55,2.6)$ \\
\hline Either crops or livestock & $14(12)$ & $3,233(7)$ & $1.9(1.1,3.3)$ \\
\hline Both crops and livestock & $6(5)$ & $826(2)$ & $3.5(1.5,8.2)$ \\
\hline
\end{tabular}

adds ratios calculated by logistic regression, adjusted for age and race/ethnicity; not calculated (NC) if 3 or fewer cases. also had an early and extended farm residence (from Table 3), so independent effects could not be assessed.

\section{DISCUSSION}

These findings support the idea that perinatal and early life exposures may influence risk of developing SLE. Ours is the first study to report SLE associations with more frequent use of pesticides at the childhood residence and with maternal/prenatal and childhood farm residence. We also saw that SLE was associated with personal exposure to agricultural pesticides in women who had lived or worked on a farm for 12 months or more up to age 18 in the absence of adult farm exposures. Together, these results suggest a dose-related elevation in SLE risk associated with early life pesticide exposure. Prior studies have suggested associations of SLE with farming occupation or personal pesticide exposures SLE (18-21), but the relevant timing of exposures was not known. These novel findings warrant replication in other populations.

We also saw associations of SLE with low birthweight and preterm birth. The independent effects of preterm birth may be difficult to identify due to the close relationship between these two factors, however, the birthweight association remained consistent when excluding preterm births. Prior evidence on birthweight and SLE is inconsistent, with one study showing SLE associated with high birthweight in women (12) and null findings in other studies $(13,24)$. Reasons for an apparent inconsistency across studies are not clear. In the prior study (combining two cohorts of the Nurses' Health Study, NHS) (12), the association with high birthweight was most apparent for the older cohort (mean age of 46 in 1976), whereas the younger cohort (mean age of 35 in 1989) showed only modest and non-significant associations of SLE with both high $(\mathrm{OR}=1.3)$ and low $(\mathrm{OR}=1.5)$ birthweight. The current study participants were more similar to the younger NHS cohort; however, cases in the NHS cohorts were incident, whereas the current study case sample included prevalent cases and could be biased if higher birthweight was related SLE severity or other reasons for non-participation. In the most recent study, linking registry data on births and SLE diagnoses in Sweden (13), the population and cases were considerably younger (average age at onset of 21 years). However, this study did not rely on recall for birthweight and had a sufficient sample size to adjust for gestational age.

Prematurity has been associated with SLE in females and younger male cases $(12,13)$. In the present study sample, a similar proportion of cases and non-cases were missing data (compared with term birth $\mathrm{OR}=1.1$; 95\%CI $0.71,1.6$ for "missingness" adjusted for age and race/ethnicity), so we did not impute missing values. Under the assumption that preterm birth is an event that is well reported if it occurs, we performed a sensitivity analysis assigning missing values to "term" birth, and results were unchanged. Many pathways and mechanisms might link birthweight and prematurity to increased risk of $\operatorname{SLE}(6,25,26)$. Limitations in the present study sample size and data precluded further investigations of life-course pathways, possibly operating through other developmental factors, such as age at menarche or adult obesity (27).

We saw no significant associations with social factors when race/ethnicity was included in the model, which is different from 
our previous findings in RA (22). Minority race/ethnicity may contribute to social disparities, and associations with young maternal age and SES score were statistically significant when only adjusted for age (not shown). We also noted that childhood SES score appeared to be associated with SLE regardless of adult educational attainment (Table S1 in Supplementary Material). Lower adult education was also associated with SLE, but we did not see any stronger association for women with both low adult education and two or more childhood SES factors as we did for RA (22). We cannot rule out the influence of selection bias. Lower SES has been associated with higher morbidity and mortality in SLE $(14,28,29)$, so one possibility is that SLE cases with, long-term lower SES and greater health disparities may have been unable to enroll in the cohort.

Few other perinatal factors were associated with SLE, including maternal or household smoking, birth season, breast feeding, being first born or number of siblings. Geographic differences in SLE across the U.S. have been previously reported, but are difficult to disentangle from racial differences $(30,31)$. In our analyses, higher latitude (proxy for lower UVB) was inversely associated with SLE risk in age-adjusted models (not shown), which was attenuated after adjusting for race/ethnicity.

We had no data on specific pesticides or types of pests treated. Organochlorines such as DDT have previously been linked to SLE risk (1). A causal association of organochlorine insecticides and SLE in susceptible individuals is supported by experimental studies, in which treatment with chlordecone, methoxychlor, or $o, p^{\prime}$-DDT accelerated SLE disease onset, including earlier increases in autoantibody levels and renal impairment in lupusprone mice, but not in a non-susceptible mouse strain. Many other pesticides impact the immune system through diverse mechanisms (32) and bear further investigation in experimental models also taking into account the question of timing relative to developmental windows of susceptibility.

Our study has several limitations, one being the use of selfreported cases. Validation studies are costly, however, and can reduce sample size and generalizability due to biased participation in studies requiring release of medical information. Because selfreport is notoriously non-specific for lupus, we increased specificity of our case definition by confirming probably clinical cases based on the self-reported use of DMARDs (33). This definition does not identify all cases, including those with incomplete medication histories or those receiving non-DMARD treatments. However, a prevalence of $\sim 2$ cases per 1,000 (e.g., 124/50,000) is reasonable for this cohort, given an estimated population prevalence of $1 / 1,000$, with higher rates in women. If socioeconomic factors and other environmental exposures are related to severity and treatment with DMARDs, then our case definition may also lead to underestimated

\section{REFERENCES}

1. Parks CG, De Roos AJ. Pesticides, chemical and industrial exposures in relation to systemic lupus erythematosus. Lupus (2014) 23:527-36. doi:10.1177/0961203313511680

2. deRosset L, Strutz KL. Developmental origins of chronic inflammation: a review of the relationship between birth weight and C-reactive protein. Ann Epidemiol (2015) 25:539-43. doi:10.1016/j.annepidem.2015.01.003 associations with disease. Disease severity may influence treatments, survival or participation in the cohort, limiting generalization of these findings to SLE in the broader population.

These analyses relied on self-reported data on perinatal and childhood factors. Sensitivity analyses were conducted, limiting the sample to women under age 60 , as reporting accuracy is expected to diminish with age and years since exposure, and younger women may be more likely to be able to consult their mothers for assistance. In most instances, we did not see changes in associations. Difficulty in reporting birthweight and preterm birth was obvious, as evidenced by the high number of missing data. Other factors may be more easily and objectively reported, such as childhood farm residence. Some immune modifying factors were not considered because they were rare (e.g., pregnancy-related hypertensive disorder or DES exposure) or not assessed (e.g., maternal nutrition) (34). Reporting of childhood pesticides may be influenced by adult pesticide use, which in turn may be influenced by health status.

Strengths of the study include the broad range of early life data available in the cohort, and results showing internally consistent findings for residential and agricultural pesticides and birthweight/prematurity. Replication of these analyses using incident cases, using a life-course approach, is warranted.

\section{AUTHOR CONTRIBUTIONS}

CP conceived of the topic, obtained, and managed the data, designed and conducted the analyses, interpreted findings, and wrote the paper. AD and DS contributed expertise on exposures, obtained and manage data, assisted with analysis design and interpreted findings, and critically reviewed the paper prior to submission. All authors agreed to final draft and submission.

\section{ACKNOWLEDGMENTS}

The authors appreciate the critical review and helpful comments of Drs Matt Longnecker and Helen Chin during preparation of the manuscript.

\section{FUNDING}

This research was supported by the Intramural Research Program of the NIH, National Institute of Environmental Health Sciences (Z01 ES044005).

\section{SUPPLEMENTARY MATERIAL}

The Supplementary Material for this article can be found online at http://journal.frontiersin.org/article/10.3389/fimmu.2016.00103 
6. Edwards CJ, Cooper C. Early environmental exposure and the development of lupus. Lupus (2006) 15:814-9. doi:10.1177/0961203306069347

7. McDade TW. Life history, maintenance, and the early origins of immune function. Am J Hum Biol (2005) 17:81-94. doi:10.1002/ajhb.20095

8. Miller GE, Chen E. The biological residue of childhood poverty. Child Dev Perspect (2013) 7:67-73. doi:10.1111/cdep.12021

9. Dietert RR. Developmental immunotoxicology (DIT): windows of vulnerability, immune dysfunction and safety assessment. J Immunotoxicol (2008) 5:401-12. doi:10.1080/15476910802483324

10. Kramer A, Bekeschus S, Bröker BM, Schleibinger H, Razavi B, Assadian O. Maintaining health by balancing microbial exposure and prevention of infection: the hygiene hypothesis versus the hypothesis of early immune challenge. J Hosp Infect (2013) 83(Suppl 1):S29-34. doi:10.1016/S0195-6701(13)60007-9

11. Schug TT, Barouki R, Gluckman PD, Grandjean P, Hanson M, Heindel JJ. PPTOX III: environmental stressors in the developmental origins of disease - evidence and mechanisms. Toxicol Sci (2013) 131:343-50. doi:10.1093/ toxsci/kfs 267

12. Simard JF, Karlson EW, Costenbader KH, Hernán MA, Stampfer MJ, Liang $\mathrm{MH}$, et al. Perinatal factors and adult-onset lupus. Arthritis Rheum (2008) 59:1155-61. doi:10.1002/art.23930

13. Arkema EV, Simard JF. Perinatal risk factors for future SLE: a population-based nested case-control study. Lupus (2015) 24:869-74. doi:10.1177/ 0961203315570160

14. Demas KL, Costenbader KH. Disparities in lupus care and outcomes. Curr Opin Rheumatol (2009) 21:102-9. doi:10.1097/BOR.0b013e328323daad

15. Eudy AM, Vines AI, Dooley MA, Cooper GS, Parks CG. Elevated C-reactive protein and self-reported disease activity in systemic lupus erythematosus. Lupus (2014) 23:1460-7. doi:10.1177/0961203314543915

16. Galobardes B, Lynch JW, Davey Smith G. Childhood socioeconomic circumstances and cause-specific mortality in adulthood: systematic review and interpretation. Epidemiol Rev (2004) 26:7-21. doi:10.1093/epirev/mxh008

17. Disanto G, Chaplin G, Morahan JM, Giovannoni G, Hyppönen E, Ebers GC, et al. Month of birth, vitamin $\mathrm{D}$ and risk of immune-mediated disease: a case control study. BMC Med (2012) 10:69. doi:10.1186/1741-7015-10-69

18. Parks CG, Walitt BT, Pettinger M, Chen JC, de Roos AJ, Hunt J, et al. Insecticide use and risk of rheumatoid arthritis and systemic lupus erythematosus in the Women's Health Initiative Observational Study. Arthritis Care Res (Hoboken) (2011) 63:184-94. doi:10.1002/acr.20335

19. Parks CG, Cooper GS, Nylander-French LA, Sanderson WT, Dement JM, Cohen PL, et al. Occupational exposure to crystalline silica and risk of systemic lupus erythematosus: a population-based, case-control study in the southeastern United States. Arthritis Rheum (2002) 46:1840-50. doi:10.1002/ art.10368

20. Gold LS, Ward MH, Dosemeci M, De Roos AJ. Systemic autoimmune disease mortality and occupational exposures. Arthritis Rheum (2007) 56:3189-201. doi:10.1002/art.22880

21. Cooper GS, Parks CG, Treadwell EL, St Clair EW, Gilkeson GS, Dooley MA. Occupational risk factors for the development of systemic lupus erythematosus. J Rheumatol (2004) 31:1928-33.

22. Parks CG, D’Aloisio AA, DeRoo LA, Huiber K, Rider LG, Miller FW, et al. Childhood socioeconomic factors and perinatal characteristics influence development of rheumatoid arthritis in adulthood. Ann Rheum Dis (2013) 72:350-6. doi:10.1136/annrheumdis-2011-201083
23. Weinberg CR, Shore DL, Umbach DM, Sandler DP. Using risk-based sampling to enrich cohorts for endpoints, genes, and exposures. Am J Epidemiol (2007) 166:447-55. doi:10.1093/aje/kwm097

24. Coleman LA, Naleway AL, Davis ME, Greenlee RT, Wilson D, McCarty DJ. Birth weight and systemic lupus erythematosus. Lupus (2005) 14:526-8. doi:10.1191/0961203305lu2152oa

25. Phillips DI. External influences on the fetus and their long-term consequences. Lupus (2006) 15:794-800. doi:10.1177/0961203306069354

26. McDade TW, Hoke M, Borja JB, Adair LS, Kuzawa C. Do environments in infancy moderate the association between stress and inflammation in adulthood? Initial evidence from a birth cohort in the Philippines. Brain Behav Immun (2013) 31:23-30. doi:10.1016/j.bbi.2012.08.010

27. Versini M, Jeandel PY, Rosenthal E, Shoenfeld Y. Obesity in autoimmune diseases: not a passive bystander. Autoimmun Rev (2014) 13:981-1000. doi:10.1016/j.autrev.2014.07.001

28. Feldman CH, Hiraki LT, Liu J, Fischer MA, Solomon DH, Alarcón GS, et al. Epidemiology and sociodemographics of systemic lupus erythematosus and lupus nephritis among US adults with Medicaid coverage, 2000-2004. Arthritis Rheum (2013) 65:753-63. doi:10.1002/art.37795

29. Fernández M, Alarcón GS, Calvo-Alén J, Andrade R, McGwin G Jr, Vilá LM, et al. A multiethnic, multicenter cohort of patients with systemic lupus erythematosus (SLE) as a model for the study of ethnic disparities in SLE. Arthritis Rheum (2007) 57:576-84. doi:10.1002/art.22672

30. Lau CS, Yin G, Mok MY. Ethnic and geographical differences in systemic lupus erythematosus: an overview. Lupus (2006) 15:715-9. doi:10.1177/0961203306072311

31. Prete PE, Majlessi A, Gilman S, Hamideh F. Systemic lupus erythematosus in men: a retrospective analysis in a Veterans Administration Healthcare System population. JClin Rheumatol (2001) 7:142-50. doi:10.1097/00124743-200106000-00003

32. Corsini E, Sokooti M, Galli CL, Moretto A, Colosio C. Pesticide induced immunotoxicity in humans: a comprehensive review of the existing evidence. Toxicology (2013) 307:123-35. doi:10.1016/j.tox.2012.10.009

33. Walitt BT, Constantinescu F, Katz JD, Weinstein A, Wang H, Hernandez RK, et al. Validation of self-report of rheumatoid arthritis and systemic lupus erythematosus: the Women's Health Initiative. J Rheumatol (2008) 35:811-8.

34. Palmer AC. Nutritionally mediated programming of the developing immune system. Adv Nutr (2011) 2:377-95. doi:10.3945/an.111.000570

Conflict of Interest Statement: The authors declare that the research was conducted in the absence of any commercial or financial relationships that could be construed as a potential conflict of interest.

This work is authored by Christine G. Parks, Aimee A. D'Aloisio and Dale P. Sandler on behalf of the U.S. Government and, as regards Dr. Parks, Dr D'Aloisio, Dr Sandler and the U.S. Government, are not subject to copyright protection in the United States. Foreign and other copyrights may apply. This is an open-access article distributed under the terms of the Creative Commons Attribution License (CC BY). The use, distribution or reproduction in other forums is permitted, provided the original author(s) or licensor are credited and that the original publication in this journal is cited, in accordance with accepted academic practice. No use, distribution or reproduction is permitted which does not comply with these terms. 